\title{
Non-Wave Variations in Temperature Caused by Sound in a Chemically Reacting Gas
}

\author{
A. Perelomova* and W. Pelc-Garska \\ Faculty of Applied Physics and Mathematics, Gdańsk University of Technology \\ G. Narutowicza 11/12, 80-233 Gdańsk, Poland
}

(Received September 27, 2010)

\begin{abstract}
A weakly nonlinear generation of non-acoustic modes in the field of sound in a gas is considered. An exoteric chemical reaction of $A \rightarrow B$ type, which takes place in a gas, may be reversible or not. Two types of sound are considered, low-frequency and high-frequency as compared with the characteristic time of a chemical reaction. For both these cases, the governing equations of non-acoustic modes are derived and conclusions of the efficiency of their nonlinear generation by sound are made. The character of nonlinear generation of non-acoustic modes by sound depends essentially on reversibility of a chemical reaction.
\end{abstract}

PACS: $43.25 .-\mathrm{x}, 43.20 . \mathrm{Bi}$

\section{Introduction}

The nonlinear losses in acoustic momentum and energy in standard thermoviscous flow result in generation of non-wave modes of a fluid motion. They are known as the thermal (or, optionally, the entropy) and vorticity modes. The latter one exists exclusively in multi-dimensional flows. Regarding to periodic sound and standard absorption in an ideal gas, these nonlinear phenomena are well-studied [1, 2]. As for flows of the non-Newtonian fluids different from ideal gases or those where dispersion of any origin takes place, the nonlinear effects of sound are still fairly poorly studied. Such fluids, however, are paid to great attention in connection to their medical and technical application. Besides the entropy and vorticity modes, there may appear other non-wave modes like it occurs in a chemically reacting fluid or a gas with excited degrees of oscillatory freedom of molecules. The important problem, among other, is how to control efficiently the mass concentration of reagents or oscillatory energy of gas molecules by means of sound.

Processes in a fluid often occur irreversibly, which leads to amplification instead of attenuation of sound. The nonlinear generation of non-acoustic modes is expected to depend strongly on reversibility of a process as well. In this study, we consider generation of the entropy and chemical modes in a gas where reversible or irreversible chemical reaction occurs. Two limiting cases of high or low-frequency sound compared with inverse time of a chemical reaction, are considered. The main idea of the method is to determine modes as relations of per-

\footnotetext{
* corresponding author; e-mail: anpe@mif.pg.gda.pl
}

turbations in a flow of infinitely-small magnitude and to fix these relations in investigations of a weakly nonlinear flow. This makes possible to distinguish dynamic equation for every mode accounting for a weak nonlinear interaction of all modes. No restriction about type of sound (periodic or not) should be done. The method was successfully used by one of the authors in studies of acoustic heating and streaming in standard absorbing fluids, some flows of non-Newtonian fluids and flows over fluids different from an ideal gas [3-5].

\section{Basic equations}

The momentum, energy, and continuity equations in a gas where a simple exoteric chemical reaction of $A \rightarrow B$ type takes place, read

$$
\begin{aligned}
& \rho \frac{\mathrm{d} \boldsymbol{v}}{\mathrm{d} t}=-\nabla P, \\
& \frac{C_{V, \infty}}{R} \frac{\mathrm{d} T}{\mathrm{~d} t}-\frac{T}{\rho} \frac{\mathrm{d} \rho}{\mathrm{d} t}=Q, \\
& \frac{\mathrm{d} \rho}{\mathrm{d} t}+\rho \nabla \cdot \boldsymbol{v}=0 .
\end{aligned}
$$

where $v, \rho, P$ denote velocity, density and pressure of a gas, respectively. In the system of Eqs. (1), $T$ is temperature measured in J per molecule, $C_{V, \infty}$ is the "frozen" heat capacity at constant volume and constant pressure, respectively (i.e., correspondent processes take place at infinitely high frequencies). $R=C_{P, \infty}-C_{V, \infty}$ is the universal gas constant, $C_{P, \infty}$ is the "frozen" heat capacity at constant pressure, $Q=H \mathrm{~mW} / \rho$ is the heat produced in the medium per one molecule due to a chemical reaction, $H$ denotes the reaction enthalpy per unit mass of reagent $A$, and $m$ denotes the averaged molecular mass of a gas. The dynamic equation of the mass fraction $Y$ 
of reagent $A$, and the equation of state complement the system (1):

$$
\frac{\mathrm{d} Y}{\mathrm{~d} t}=-\frac{W}{\rho}, \quad P=\frac{\rho T}{m} .
$$

\section{Dispersion relations in one-dimensional flow}

The one-dimensional gas flow along axis $O X$ is considered. Every quantity in Eqs. (1) represents sum of unperturbed value and its variation, for example: $T=T_{0}+T^{\prime}$ (where in a weakly nonlinear flow $\left|T^{\prime}\right| \ll T_{0}$, and so on). Following $[6,7]$, we assume that the stationary quantities $Y_{0}, T_{0}, P_{0}, \rho_{0}, v_{0}=0$ are maintained by a transverse pumping, so that in the longitudinal direction pointed by axis $O X$, the stationary medium is homogeneous. Equations (1), with account for Eq. (2) within accuracy of quadratic nonlinear terms, read

$$
\begin{aligned}
& \frac{\partial v^{\prime}}{\partial t}+\frac{T_{0}}{m \rho_{0}} \frac{\partial \rho^{\prime}}{\partial x}+\frac{1}{m} \frac{\partial T^{\prime}}{\partial x} \\
& =-v^{\prime} \frac{\partial v^{\prime}}{\partial x}+\frac{T_{0} \rho^{\prime}}{m \rho_{0}^{2}} \frac{\partial \rho^{\prime}}{\partial x}-\frac{T^{\prime}}{m \rho_{0}} \frac{\partial \rho^{\prime}}{\partial x}, \\
& \frac{\partial T^{\prime}}{\partial t}+\left(\gamma_{\infty}-1\right)\left(T_{0} \frac{\partial v^{\prime}}{\partial x}-Q_{T} \frac{Q_{0}}{T_{0}} T^{\prime}-Q_{\rho} \frac{Q_{0}}{\rho_{0}} \rho^{\prime}\right. \\
& \left.-Q_{Y} \frac{Q_{0}}{Y_{0}} Y^{\prime}\right)=-v^{\prime} \frac{\partial T^{\prime}}{\partial x}-\left(\gamma_{\infty}-1\right) T^{\prime} \frac{\partial v^{\prime}}{\partial x}, \\
& \frac{\partial Y^{\prime}}{\partial t}+\frac{1}{H m}\left(Q_{T} \frac{Q_{0}}{T_{0}} T^{\prime}+Q_{\rho} \frac{Q_{0}}{\rho_{0}} \rho^{\prime}+Q_{Y} \frac{Q_{0}}{Y_{0}} Y^{\prime}\right) \\
& =-v^{\prime} \frac{\partial Y^{\prime}}{\partial x}, \\
& \frac{\partial \rho^{\prime}}{\partial t}+\rho_{0} \frac{\partial v^{\prime}}{\partial x}=-v^{\prime} \frac{\partial \rho^{\prime}}{\partial x}-\rho^{\prime} \frac{\partial v^{\prime}}{\partial x},
\end{aligned}
$$

where $\gamma_{\infty}=\frac{C_{P, \infty}}{C_{V, \infty}}$ denotes the frozen adiabatic exponent and dimensionless quantities $Q_{T}, Q_{\rho}, Q_{Y}$ depend on the heat produced due to a chemical reaction

$$
\begin{aligned}
Q_{T} & =\frac{T_{0}}{Q_{0}}\left(\frac{\partial Q}{\partial T}\right)_{T_{0}, \rho_{0}, Y_{0}}, \quad Q_{\rho}=\frac{\rho_{0}}{Q_{0}}\left(\frac{\partial Q}{\partial \rho}\right)_{T_{0}, \rho_{0}, Y_{0}}, \\
Q_{Y} & =\frac{Y_{0}}{Q_{0}}\left(\frac{\partial Q}{\partial Y}\right)_{T_{0}, \rho_{0}, Y_{0}} .
\end{aligned}
$$

The linearized version of Eq. (3) describes a flow of infinitely-small magnitude

$$
\begin{aligned}
& \frac{\partial v^{\prime}}{\partial t}+\frac{T_{0}}{m \rho_{0}} \frac{\partial \rho^{\prime}}{\partial x}+\frac{1}{m} \frac{\partial T^{\prime}}{\partial x}=0 \\
& \frac{\partial T^{\prime}}{\partial t}+\left(\gamma_{\infty}-1\right)\left(T_{0} \frac{\partial v^{\prime}}{\partial x}-Q_{T} \frac{Q_{0}}{T_{0}} T^{\prime}-Q_{\rho} \frac{Q_{0}}{\rho_{0}} \rho^{\prime}\right. \\
& \left.\quad-Q_{Y} \frac{Q_{0}}{Y_{0}} Y^{\prime}\right)=0
\end{aligned}
$$

$$
\begin{aligned}
& \frac{\partial Y^{\prime}}{\partial t}+\frac{1}{H m}\left(Q_{T} \frac{Q_{0}}{T_{0}} T^{\prime}+Q_{\rho} \frac{Q_{0}}{\rho_{0}} \rho^{\prime}+Q_{Y} \frac{Q_{0}}{Y_{0}} Y^{\prime}\right)=0 \\
& \frac{\partial \rho^{\prime}}{\partial t}+\rho_{0} \frac{\partial v^{\prime}}{\partial x}=0 .
\end{aligned}
$$

Let any perturbation represent a sum of planar waves proportional to $\exp (\mathrm{i} \omega t-\mathrm{i} k x)$. Every type of motion is determined by one of the root of dispersion relation of the linear flow, $\omega(k)$. From the third equation from the system (5) the equalities below follow:

$$
\begin{aligned}
& \left(\frac{\partial Y}{\partial T}\right)_{V}=-\frac{Q_{T}}{Q_{Y}\left(1+\mathrm{i} \omega \tau_{\mathrm{c}}\right)} \frac{Y_{0}}{T_{0}}, \\
& \left(\frac{\partial Y}{\partial \rho}\right)_{T}=-\frac{1}{\rho_{0}^{2}}\left(\frac{\partial Y}{\partial V}\right)_{T}=-\frac{Q_{\rho}}{Q_{Y}\left(1+\mathrm{i} \omega \tau_{\mathrm{c}}\right)} \frac{Y_{0}}{\rho_{0}},
\end{aligned}
$$

where $V=1 / \rho$ designates the specific gas volume, and

$$
\tau_{\mathrm{c}}=\frac{H m Y_{0}}{Q_{0} Q_{Y}}
$$

is the characteristic time of chemical reaction. The equation of state (the second from Eqs. (2)) along with the thermodynamic equality

$$
\left(\frac{\partial Y}{\partial T}\right)_{P}=\left(\frac{\partial Y}{\partial T}\right)_{V}+\left(\frac{\partial Y}{\partial V}\right)_{T}\left(\frac{\partial V}{\partial T}\right)_{P},
$$

result in the following expression:

$$
\left(\frac{\partial Y}{\partial T}\right)_{P}=\frac{\left(Q_{\rho}-Q_{T}\right)}{Q_{Y}\left(1+\mathrm{i} \omega \tau_{\mathrm{c}}\right)} \frac{Y_{0}}{T_{0}}
$$

The dispersion relations for three non-thermal modes (two acoustic and one non-wave), and the thermal one, are described by equalities

$$
\begin{aligned}
\omega^{2} & =k^{2} \frac{T_{0}}{m} \frac{C_{P}}{C_{V}}=k^{2} \frac{T_{0}}{m}\left(\frac{C_{P, \infty}+m H\left(\frac{\partial Y}{\partial T}\right)_{P}}{C_{V, \infty}+m H\left(\frac{\partial Y}{\partial T}\right)_{V}}\right) \\
& =k^{2} \frac{T_{0}}{m}\left(\frac{C_{P, \infty}+\frac{\left(Q_{\rho}-Q_{T}\right) \tau_{\mathrm{c}}}{1+\mathrm{i} \omega \tau_{\mathrm{c}}} \frac{Q_{0}}{T_{0}}}{C_{V, \infty}-\frac{Q_{T} \tau_{c}}{1+\mathrm{i} \omega \tau_{\mathrm{c}}} \frac{Q_{0}}{T_{0}}}\right), \quad \omega=0 .
\end{aligned}
$$

The approximate roots of dispersion relations for both acoustic branches, were firstly derived in [7]. It is reasonable to evaluate first three roots of dispersion equation in two opposite cases: when acoustic frequency is large compared with the characteristic duration of chemical reaction $\tau_{\mathrm{c}}: \omega \tau_{\mathrm{c}} \approx|k| u_{\infty} \tau_{\mathrm{c}} \gg 1$ and when acoustic frequency is small compared with $\tau_{\mathrm{c}}: \omega \tau_{\mathrm{c}} \approx|k| u_{0} \tau_{\mathrm{c}} \ll 1$ $\left(u_{\infty}, u_{0}\right.$ are sound velocity of infinitely large and small frequency, respectively). In both cases a slow dependence of heat release in a chemical reaction on temperature and density $\left(\left|Q_{\rho}\right| \ll 1,\left|Q_{T}\right| \ll 1\right)$ is assumed.

\section{High-frequency domain}

In this section the high-frequency domain of sound frequencies is considered: $\omega \tau_{\mathrm{c}} \gg 1$. Regarding to the third, non-wave mode, this condition determines the characteristic wave numbers, $|k| u_{\infty} \tau_{\mathrm{c}} \gg 1$ :

$$
\omega_{1}=u_{\infty}\left(k-\mathrm{i} B_{\infty}\right), \quad \omega_{2}=u_{\infty}\left(-k-\mathrm{i} B_{\infty}\right),
$$




$$
\omega_{3}=\mathrm{i}\left[\frac{1}{\tau_{\mathrm{c}}}+\frac{\left(\gamma_{\infty}-1\right) Q_{0}\left(Q_{\rho}-Q_{T}\right)}{u_{\infty}^{2} m}\right], \quad \omega_{4}=0,
$$

where

$$
B_{\infty}=\frac{Q_{0}\left(\gamma_{\infty}-1\right)\left[Q_{\rho}+\left(\gamma_{\infty}-1\right) Q_{T}\right]}{2 u_{\infty}^{3} m}
$$

and $u_{\infty}=\sqrt{\gamma_{\infty} \frac{T_{0}}{m}}$ is the frozen sound velocity. The first two roots in (11): $\omega_{1}, \omega_{2}$ are acoustic and $\omega_{4}$ corresponds to the thermal (entropy) mode. The third non-acoustic root $\omega_{3}$ is responsible for the non-wave variation in mass fraction of reagent $A$. For sound to be a wave process, attenuation (when $B_{\infty}$ is negative, or amplification, if $B_{\infty}$ is positive) should be small compared with the characteristic acoustic wave number, $\left|B_{\infty}\right| \ll|k|$. This in fact determines smallness of $\left|Q_{\rho}\right|,\left|Q_{T}\right|$ more precisely in dependence on the characteristic domain of sound wave numbers.

\subsection{Decomposition of equations}

4.1.1. Linear equations governing acoustic and nonacoustic modes

Four dispersion relations (11) determine four independent modes: $\psi=\psi_{1}+\psi_{2}+\psi_{3}+\psi_{4}=\left(v^{\prime}, T^{\prime}, Y^{\prime}, \rho^{\prime}\right)^{\mathrm{T}}$. In general, every perturbation of the field variables contains contributions from each of the four modes, for example, $\rho^{\prime}=\rho_{1}^{\prime}+\rho_{2}^{\prime}+\rho_{3}^{\prime}+\rho_{4}^{\prime}$. This allows decomposition equations governing every mode in their linear parts using specific properties of modes. Taking in mind that $(-\mathrm{i} k)^{-1}$ corresponds to the operator $\int \mathrm{d} x$, one may derive the modes,

$$
\begin{aligned}
& \psi_{1}=\left(\begin{array}{c}
v_{1}^{\prime} \\
T_{1}^{\prime} \\
Y_{1}^{\prime} \\
\rho_{1}^{\prime}
\end{array}\right)=\left(\begin{array}{c}
\frac{u_{\infty}}{\rho_{0}}-\frac{u_{\infty} B_{\infty}}{\rho_{0}} \int \mathrm{d} x \\
\frac{\left(\gamma_{\infty}-1\right) T_{0}}{\rho_{0}}-\frac{2 \gamma_{\infty} T_{0} B_{\infty}}{\rho_{0}} \int \mathrm{d} x \\
\frac{2 B_{\infty} u_{\infty}^{2}}{\left(\gamma_{\infty}-1\right) H \rho_{0}} \int \mathrm{d} x \\
1
\end{array}\right) \rho_{1}^{\prime}, \\
& \psi_{2}=\left(\begin{array}{c}
-\frac{u_{\infty}}{\rho_{0}}-\frac{u_{\infty} B_{\infty}}{\rho_{0}} \int \mathrm{d} x \\
\frac{\left(\gamma_{\infty}-1\right) T_{0}}{\rho_{0}}+\frac{2 \gamma_{\infty} T_{0} B_{\infty}}{\rho_{0}} \int \mathrm{d} x \\
-\frac{2 B_{\infty} u_{\infty}^{2}}{\left(\gamma_{\infty}-1\right) H \rho_{0}} \int \mathrm{d} x \\
1
\end{array}\right) \rho_{2}^{\prime}, \\
& \psi_{3}=\left(\begin{array}{c}
\left(\frac{\left(\gamma_{\infty}-1\right) Q_{0}\left(Q_{\rho}-Q_{T}\right)}{\rho_{0} T_{0} \gamma_{\infty}}+\frac{1}{\tau_{\mathrm{c}} \rho_{0}}\right) \int \mathrm{d} x \\
-\frac{T_{0}}{\rho_{0}} \\
\frac{u_{\infty}^{2}}{H\left(\gamma_{\infty}-1\right) \rho_{0}} \\
1
\end{array}\right) \rho_{3}^{\prime}, \\
& \psi_{4}=\left(\begin{array}{c}
0 \\
-\frac{T_{0}}{\rho_{0}} \\
-\frac{\tau_{\mathrm{c}} Q_{0}\left(Q_{\rho}-Q_{T}\right)}{H m \rho_{0}} \\
1
\end{array}\right) \rho_{4}^{\prime} .
\end{aligned}
$$

The linear equations for any mode may be decomposed directly from the system (5). The dynamic equation governing an excess density in acoustic wave progressive in the positive direction of axis $O X$, and these for the entropy and the chemical modes are as follows:

$$
\begin{aligned}
& \frac{\partial \rho_{1}^{\prime}}{\partial t}+u_{\infty} \frac{\partial \rho_{1}^{\prime}}{\partial x}-B_{\infty} \rho_{1}^{\prime}=0, \\
& \frac{\partial \rho_{4}^{\prime}}{\partial t}=0, \\
& \frac{\partial \rho_{3}^{\prime}}{\partial t}+\left[\frac{Q_{0}\left(\gamma_{\infty}-1\right)\left(Q_{\rho}-Q_{T}\right)}{T_{0} \gamma_{\infty}}+\frac{1}{\tau_{\mathrm{c}}}\right] \rho_{3}^{\prime}=0 .
\end{aligned}
$$

Equations (14) coincide with the roots of dispersion equation, Eqs. (11).

\subsubsection{Non-linear corrections in acoustic mode}

The linear links specific for sound should be complemented by second-order non-linear terms making it isentropic in the leading order. In this study, we consider nonlinear terms of order not higher than second with respect to powers of perturbation of excess density. For simplicity, sound will be associated with the wave propagating in the positive direction of axis $O X$. The corrected links specific for the first acoustic mode are

$$
\begin{aligned}
v_{1}^{\prime} & =\frac{u_{\infty}}{\rho_{0}} \rho_{1}^{\prime}-\frac{u_{\infty} B_{\infty}}{\rho_{0}} \int \rho_{1}^{\prime} \mathrm{d} x+\frac{\left(\gamma_{\infty}-3\right) u_{\infty}}{4 \rho_{0}^{2}} \rho_{1}^{\prime 2}, \\
T_{1}^{\prime} & =\frac{\left(\gamma_{\infty}-1\right) T_{0}}{\rho_{0}} \rho_{1}^{\prime}-\frac{2 u_{\infty}^{2} m B_{\infty}}{\rho_{0}} \int \rho_{1}^{\prime} \mathrm{d} x \\
& +\frac{\left(\gamma_{\infty}-1\right)\left(\gamma_{\infty}-2\right) T_{0}}{2 \rho_{0}^{2}} \rho_{1}^{\prime 2} .
\end{aligned}
$$

In fact, the nonlinear corrections in Eqs. (15) establish relationships specific for the planar Riemann wave $[1,8]$ propagating over an ideal gas. Taking into account non-linear terms in the governing equation for sound, one may readily conclude that an excess acoustic density satisfies an equation

$$
\frac{\partial \rho_{1}^{\prime}}{\partial t}+u_{\infty} \frac{\partial \rho_{1}^{\prime}}{\partial x}+\frac{\left(\gamma_{\infty}+1\right) u_{\infty}}{2 \rho_{0}} \rho_{1}^{\prime} \frac{\partial \rho_{1}^{\prime}}{\partial x}-B_{\infty} \rho_{1}^{\prime}=0,
$$

which looks similarly to the Earnshaw equation [1] but includes the term $-B_{\infty} \rho_{1}^{\prime}$ responsible for attenuation or amplification of sound in dependence on sign of $B_{\infty}$.

\subsubsection{Variations in temperature caused by high-frequency sound}

We assume magnitudes of both non-wave modes small as compared with that of sound, $\left|T_{3}^{\prime}\right|,\left|T_{4}^{\prime}\right| \ll \max \left|T_{1}^{\prime}\right|$. The variations in temperature of the non-wave modes form a new background of a medium. That is why studies of non-linear generation of non-wave modes by sound may be of great importance. Multiplying the first equation from system (3) by 0 and the second, third and fourth equations by corresponding factors

$$
\begin{aligned}
& -\frac{\rho_{0}}{m u_{\infty}^{2}}+\frac{\rho_{0} Q_{0}\left(Q_{\rho}-Q_{T}\right)\left(\gamma_{\infty}-1\right) \tau_{\mathrm{c}}}{m^{2} u_{\infty}^{4}} \\
& \frac{\rho_{0} H\left(\gamma_{\infty}-1\right)\left(-m u_{\infty}^{2}+Q_{0}\left(Q_{\rho}-Q_{T}\right)\left(\gamma_{\infty}-1\right) \tau_{\mathrm{c}}\right)}{m u_{\infty}^{4}}
\end{aligned}
$$




$$
1-\frac{1}{\gamma_{\infty}}-\frac{\left(\gamma_{\infty}-1\right)^{2} Q_{0}\left(Q_{\rho}-Q_{T}\right) \tau_{\mathrm{c}}}{m u_{\infty}^{2} \gamma_{\infty}},
$$

respectively, and taking a sum of all equations reduces all terms belonging to the first, second and third modes in the linear part of the final equation. As for the nonlinear part of the final equation, only acoustic quadratic terms are considered there. That yields the equation for an excess temperature attributable to the entropy mode

$$
\frac{\partial T_{4}^{\prime}}{\partial t}=2 u_{\infty}\left(\gamma_{\infty}-1\right) B_{\infty} \frac{T_{0}}{\rho_{0}^{2}} \frac{\partial \rho_{1}^{\prime}}{\partial x} \int \rho_{1}^{\prime} \mathrm{d} x .
$$

Equation (17) describes heating or cooling generated by an acoustic source which represents the right-hand side of it. In order to reduce all terms besides that belonging to the third mode in the linear part, one should multiply the first equation of system (3) by the factor

$$
\frac{Q_{0}\left(\gamma_{\infty}-1\right)\left[Q_{\rho}+\left(\gamma_{\infty}-1\right) Q_{T}\right]}{\gamma_{\infty} u_{\infty}^{2}} \int \mathrm{d} x,
$$

and the second, third and fourth equations by factors

$$
\begin{aligned}
& \frac{\tau_{\mathrm{c}} Q_{0}\left(\gamma_{\infty}-1\right)\left(Q_{T}-Q_{\rho}\right)}{\gamma_{\infty}^{2} T_{0}}, \\
& -\frac{H m\left(\gamma_{\infty}-1\right)}{\gamma_{\infty}}-\frac{\tau_{\mathrm{c}} Q_{0} H\left(\gamma_{\infty}-1\right)^{2}\left(Q_{T}-Q_{\rho}\right)}{\gamma_{\infty} u_{\infty}^{2}}, \\
& \frac{\tau_{\mathrm{c}} Q_{0}\left(\gamma_{\infty}-1\right)^{2}\left(Q_{T}-Q_{\rho}\right)}{\gamma_{\infty}^{2} \rho_{0}},
\end{aligned}
$$

respectively. Then taking a sum of all the equations and keeping only acoustic quadratic terms, result finally in the equation

$$
\begin{aligned}
& \frac{\partial T_{3}^{\prime}}{\partial t}+\left(\frac{1}{\tau_{\mathrm{c}}}+\frac{Q_{0}\left(\gamma_{\infty}-1\right)\left(Q_{\rho}-Q_{T}\right)}{\gamma_{\infty} T_{0}}\right) T_{3}^{\prime} \\
& =-\frac{Q_{0}\left(\gamma_{\infty}-1\right)\left[\left(\gamma_{\infty}-3\right) Q_{\rho}-\left(\gamma_{\infty}-1\right)^{2} Q_{T}\right]}{4 \gamma_{\infty} \rho_{0}^{2}} \rho_{1}^{\prime 2} .
\end{aligned}
$$

It describes an excess temperature attributable to the non-acoustic third (chemical) mode.

\section{Low-frequency domain}

In this section, dispersion relations, modes and all relative equations will be calculated in the similar way as in Sect. (4), but under the following assumption: $\omega \tau_{\mathrm{c}} \approx|k| u_{0} \tau_{\mathrm{c}} \ll 1$. The leading-order dispersion relations in the low-frequency case are

$$
\begin{aligned}
& \omega_{1}=u_{0}\left(k-\mathrm{i} B_{0}\right), \quad \omega_{2}=u_{0}\left(-k-\mathrm{i} B_{0}\right), \\
& \omega_{3}=\frac{\mathrm{i}}{\tau_{\mathrm{c}}}-\frac{\mathrm{i} R Q_{0} Q_{T}}{C_{V, 0} T_{0}}, \quad \omega_{4}=0,
\end{aligned}
$$

where

$$
B_{0}=\frac{k^{2} R \tau_{\mathrm{c}}^{2} Q_{0}\left[Q_{\rho}+\left(\gamma_{0}-1\right) Q_{T}\right] u_{0}}{2 C_{P, 0} T_{0}},
$$

$$
\begin{aligned}
& C_{P, 0}=C_{P, \infty}+\frac{R Q_{0} \tau_{\mathrm{c}}\left(Q_{\rho}-Q_{T}\right)}{T_{0}}, \\
& C_{V, 0}=C_{V, \infty}-\frac{R Q_{0} \tau_{\mathrm{c}} Q_{T}}{T_{0}}
\end{aligned}
$$

are the equilibrium (low-frequency) heat capacities, $u_{0}=$ $\sqrt{\gamma_{0} \frac{T_{0}}{m}}$ is an infinitely small signal velocity, and

$$
\gamma_{0}=\frac{C_{P, 0}}{C_{V, 0}} \approx \gamma_{\infty}+\frac{2 u_{\infty} B_{\infty} \tau_{\mathrm{c}}}{\gamma_{\infty}} .
$$

\subsection{Decomposition of equations}

\subsubsection{Linear equations governing acoustic} and non-acoustic modes

The dispersion relations Eqs. (19) corresponding to the linear equations describe the dynamics of an excess density in acoustic mode (propagating in the positive direction of axis $O X$ ) and this of the non-acoustic modes, respectively

$$
\begin{aligned}
& \frac{\partial \rho_{1}^{\prime}}{\partial t}+u_{0} \frac{\partial \rho_{1}^{\prime}}{\partial x}=0, \quad \frac{\partial \rho_{4}^{\prime}}{\partial t}=0, \\
& \frac{\partial \rho_{3}^{\prime}}{\partial t}+\left(\frac{1}{\tau_{\mathrm{c}}}-\frac{R Q_{0} Q_{T}}{C_{V, 0} T_{0}}\right) \rho_{3}^{\prime}=0 .
\end{aligned}
$$

\subsubsection{Non-linear corrections in acoustic mode}

Analogically to Sect. 4, the linear relation in low-frequency sound modes should be complemented by the second-order terms, making these modes isentropic in the leading order

$$
\begin{aligned}
& v_{1}^{\prime}=\frac{u_{0}}{\rho_{0}} \rho_{1}^{\prime}+\frac{u_{0}\left(\gamma_{0}-3\right)}{4 \rho_{0}^{2}} \rho_{1}^{\prime 2}, \\
& T_{1}^{\prime}=\frac{m u_{0}^{2}\left(\gamma_{0}-1\right)}{\gamma_{0} \rho_{0}} \rho_{1}^{\prime}+\frac{\left(\gamma_{0}-1\right)\left(\gamma_{0}-2\right) T_{0}}{2 \rho_{0}^{2}} \rho_{1}^{\prime 2} .
\end{aligned}
$$

The weakly nonlinear equation governing an excess acoustic density, takes a form of the Earnshaw equation [1], because attenuation of a low-frequency sound is negligible

$$
\frac{\partial \rho_{1}^{\prime}}{\partial t}+u_{0} \frac{\partial \rho_{1}^{\prime}}{\partial x}+\frac{\left(\gamma_{0}+1\right) u_{0}}{2 \rho_{0}} \rho_{1}^{\prime} \frac{\partial \rho_{1}^{\prime}}{\partial x}=0 .
$$

5.1.3. Variations in temperature caused by low-frequency sound

To obtain the equation of an excess temperature of the entropy mode in the low-frequency domain, the first equation from the system (3) should be multiplied by 0 , and the second, third and fourth equations by the following factors:

$$
-\frac{\rho_{0}}{T_{0} \gamma_{0}}-\frac{\rho_{0} Q_{0} Q_{T} R \tau_{\mathrm{c}}}{C_{P 0} T_{0}^{2}}, \quad-\frac{\rho_{0} H m R}{T_{0} C_{P 0}}, \quad \frac{R}{C_{P 0}},
$$

respectively. Taking the sum of all equations reduces terms attributable to the first, second and third modes in the linear part of the resulting equation. The equation governing the acoustic heating (or cooling in dependence on sign of the right-hand side of equation) in the leading order takes the form 


$$
\begin{aligned}
& \frac{\partial T_{4}^{\prime}}{\partial t}=-\frac{R \tau_{\mathrm{c}} Q_{0} u_{0}\left[\left(\gamma_{0}+3\right) Q_{\rho}+\left(\gamma_{0}-1\right)\left(\gamma_{0}-3\right) Q_{T}\right]}{2 \rho_{0}^{2} C_{P, 0}} \\
& \quad \times \rho_{1}^{\prime} \frac{\partial \rho_{1}^{\prime}}{\partial x} .
\end{aligned}
$$

It is readily concluded that generation of the entropy mode by the low-frequency sound (both periodic or aperiodic) is inefficient, because the acoustic source is of order $\max \left(\left|Q_{\rho}\right|,\left|Q_{T}\right|\right) \times|k| u_{0} \tau_{\mathrm{c}}$. Moreover, the term $\rho_{1}^{\prime} \frac{\partial \rho_{1}^{\prime}}{\partial x}$ for the periodic waveform proportional to $\sin \left(\omega\left(t-x / u_{0}\right)\right)$ would result 0 after averaging over the sound period $2 \pi / \omega$.

As for the third mode, one can readily obtain the dynamic equation for it by multiplying the first equation of the system (3) by 0 and the second, third and fourth equations by the factors

$$
-\frac{R \tau_{\mathrm{c}} Q_{0} Q_{T}}{C_{V, 0} T_{0}}, \quad-\frac{R H m}{C_{V, 0}}, \quad-\frac{R \tau_{\mathrm{c}} Q_{0} Q_{\rho}}{C_{V, 0} \rho_{0}},
$$

respectively, and taking a sum of all equations. The resulting equation reads

$$
\begin{aligned}
& \frac{\partial T_{3}^{\prime}}{\partial t}+\left(\frac{1}{\tau_{\mathrm{c}}}-\frac{R Q_{0} Q_{T}}{C_{V, 0} T_{0}}\right) T_{3}^{\prime} \\
& =\frac{Q_{T} Q_{0}\left(\gamma_{0}-1\right)^{2}\left(\gamma_{0}-2\right)}{2 \rho_{0}^{2}} \rho_{1}^{\prime 2} .
\end{aligned}
$$

\section{Illustrations}

\subsection{Variations in temperature attributable to the entropy mode generated by periodic high-frequency sound} form

Equation (17) in dimensionless quantities takes the

$$
\frac{\partial T_{4}^{\prime}}{\partial(\omega t)}=2\left(\gamma_{\infty}-1\right) B_{\infty}^{*} \frac{T_{0}}{\rho_{0}^{2}} \frac{\partial \rho_{1}^{\prime}}{\partial x^{*}} \int \rho_{1}^{\prime} \mathrm{d} x^{*},
$$

where $B_{\infty}^{*}=B_{\infty} u_{\infty} / \omega, x^{*}=\omega x / u_{\infty}$ are the dimensionless quantities. An excess acoustic density itself satisfies nonlinear Eq. (16). The solution of it, correspondent to the periodic on the transducer sound $\rho_{1}^{\prime}(x=0, t)=$ $M \rho_{0} \sin (\omega t)$, is represented by the sum

$$
\begin{aligned}
& \rho_{1}^{\prime}(x, t)=M \rho_{0} \exp \left(B_{\infty}^{*} x^{*}\right) \\
& \quad \times \sum_{n=1}^{\infty} \frac{2 J_{n}\left[n K\left(\exp \left(B_{\infty}^{*} x^{*}\right)-1\right)\right] \sin \left(n \theta_{\infty}\right)}{n K\left[\exp \left(B_{\infty}^{*} x^{*}\right)-1\right]},
\end{aligned}
$$

where

$$
K=\frac{\left(\gamma_{\infty}+1\right) M}{2 B_{\infty}^{*}}, \quad \theta_{\infty}=\omega\left(t-x / u_{\infty}\right) .
$$

$M$ denotes the acoustic Mach number. Equation (28) is valid at distances from transducer smaller than the characteristic distance of wave breakup, $x_{\mathrm{b}}$ :

$$
x_{\mathrm{b}}=\frac{1}{B_{\infty}^{*}} \ln (1+1 / K) .
$$

In view of that the coefficient $B_{\infty}^{*}$ is a small parameter as compared with the acoustic wave number $k$, it is reasonable to evaluate the integral of the following type approximately:

$$
\int f\left(B_{\infty}^{*} x^{*}\right) F\left(\theta_{\infty}\right) \mathrm{d} x^{*} \approx f\left(B_{\infty}^{*} x^{*}\right) \int F\left(\theta_{\infty}\right) \mathrm{d} x^{*},
$$

and to consider the acoustic source in Eq. (27) averaged over the sound period. The resulting averaged temporal derivative of temperature, associated with the thermal mode, will be exclusively function of $x$. The variation in temperature $\left\langle\partial\left(T^{\prime} / T_{0}\right) / \partial(\omega t)\right\rangle$ for different coefficients $B_{\infty}^{*}$ is shown in Fig. 1 for some positive and negative values of $B_{\infty}^{*}$. The angle brackets denote averaging over the sound period, $\langle f\rangle=\frac{\omega}{2 \pi} \int_{t}^{t+2 \pi / \omega} f \mathrm{~d} t$. To compute variation in temperature, the following coefficients have been used: $M=0.01, \gamma_{\infty}=1.4$. One may conclude

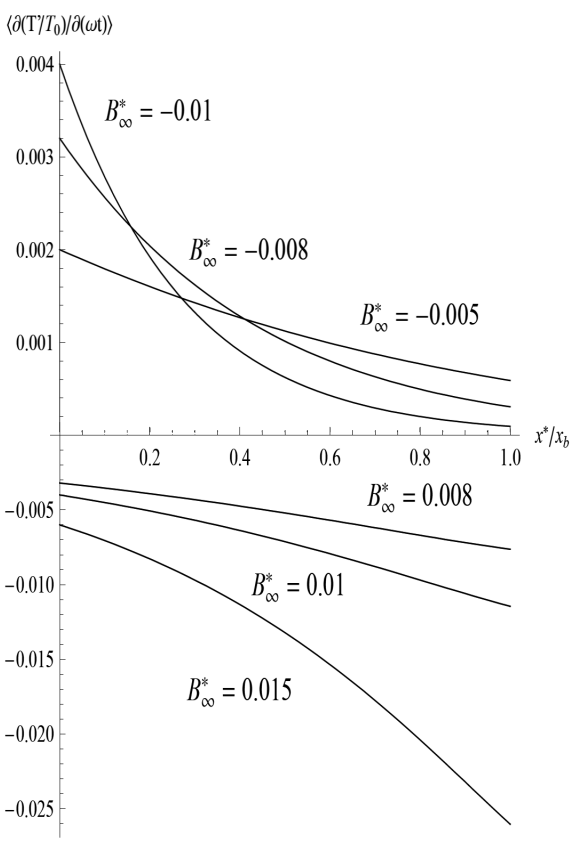

Fig. 1. The dimensionless variation in temperature for coefficients $B_{\infty}^{*}$, respectively.

from Fig. 1 that the ambient temperature increases in time, when coefficient $B_{\infty}^{*}$ is negative, and decreases otherwise. The rate of increase (or decrease) in temperature depends on value of $B_{\infty}^{*}$ and distance from a transducer. The acoustic energy in the case of negative $B_{\infty}^{*}$ decreases, and nonlinear attenuation of sound results in generation of the thermal mode like it occurs in standard thermoviscous fluids. However, $B_{\infty}^{*}>0$ provides the acoustic cooling. Possibility of acoustic cooling instead of heating was firstly pointed out by Molevich [9] regarding to different dispersive flow over a gas with excited irreversibly degrees of freedom of molecules.

\subsection{Variations in temperature attributable to the entropy mode generated by pulses of equal energy}

The most simple acoustic waveform is this one propagating with constant velocity $u_{\infty}$ without change of its shape. It may be considered as an approximate solution of Eq. (16) (when one neglects two last terms in it, responsible for nonlinearity and attenuation, or 
amplification of sound). To compare nonlinear generation of the entropy mode of three acoustic pulses of equal energy, the following waveforms will be considered, numbered by 1,2 , and 3 , respectively:

$$
\begin{aligned}
& \rho_{1}^{\prime}=M \rho_{0} \exp \left(-\theta_{\infty}^{2} / 0.1^{2}\right), \\
& \rho_{2}^{\prime}=1.8857 M \rho_{0} \theta_{\infty}^{2} / 0.15^{2} \exp \left(-\theta_{\infty}^{2} / 0.15^{2}\right), \\
& \rho_{3}^{\prime}=1.414 M \rho_{0} \theta_{\infty} / 0.2 \exp \left(-\theta_{\infty}^{2} / 0.2^{2}\right) .
\end{aligned}
$$

Energy of all three pulses, proportional to $\int_{-\infty}^{\infty}\left(\rho^{\prime}\left(\theta_{\infty}\right)\right)^{2} \mathrm{~d} \theta_{\infty}$, is equal. The initial waveforms are shown in Fig. 2a. An increase in the temperature for $B_{\infty}^{*}=-0.005$ in accordance to Eq. (27) is shown in Fig. 2b. The numerical simulations reveal that an

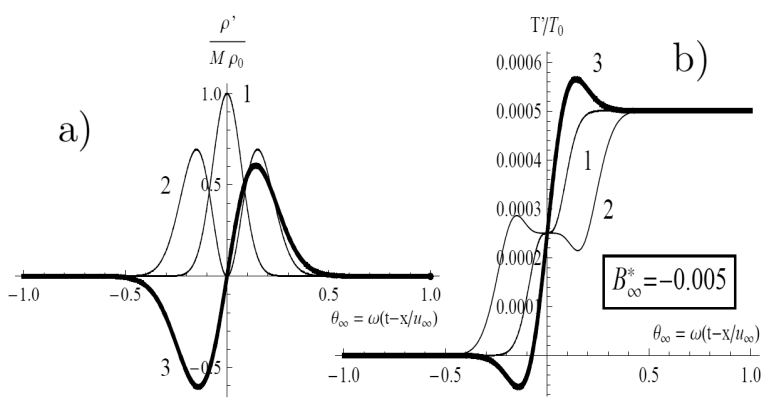

Fig. 2. (a) The initial waveforms in accordance to Eq. (32) and (b) the dimensionless ambient excess temperature caused by these three waveforms when $B_{\infty}^{*}=$ -0.005 .

efficiency of acoustic heating (or cooling) does not depend on waveforms associated to the entropy mode if the energy of waves is the same, independently of a sign of $B_{\infty}^{*}$. However, in media with different relaxation process, for example the Maxwell relaxation taking place in viscoelastic biological media, efficiency of acoustic heating may depend on the sound waveform [10].

\subsection{Variations in temperature attributable to the chemical mode}

Both Eqs. (18), (26), describing effects of low-frequency or high-frequency sound, have the form

$$
\frac{\partial T_{3}^{\prime}}{\partial t}+\beta T_{3}^{\prime}=\alpha T_{0} \frac{\rho_{1}^{\prime 2}}{\rho_{0}^{2}},
$$

where in the low-frequency domain of sound frequencies,

$$
\begin{aligned}
& \beta=\beta_{0}=\frac{1}{\tau_{\mathrm{c}}}-\frac{R Q_{0} Q_{T}}{C_{V, 0} T_{0}} \approx \frac{1}{\tau_{\mathrm{c}}}-\frac{Q_{0} Q_{T}\left(\gamma_{0}-1\right)}{T_{0}}, \\
& \alpha=\alpha_{0}=\frac{Q_{T} Q_{0}\left(\gamma_{0}-1\right)^{2}\left(\gamma_{0}-2\right)}{2 T_{0}},
\end{aligned}
$$

and for the high-frequency sound,

$$
\begin{aligned}
& \beta=\beta_{\infty}=\frac{1}{\tau_{\mathrm{c}}}+\frac{Q_{0}\left(\gamma_{\infty}-1\right)\left(Q_{\rho}-Q_{T}\right)}{\gamma_{\infty} T_{0}}, \\
& \alpha=\alpha_{\infty}
\end{aligned}
$$

$$
=-\frac{Q_{0}\left(\gamma_{\infty}-1\right)\left[\left(\gamma_{\infty}-3\right) Q_{\rho}-\left(\gamma_{\infty}-1\right)^{2} Q_{T}\right]}{4 \gamma_{\infty} T_{0}} .
$$

Equation (33) is readily integrated

$$
T_{3}^{\prime}=\frac{\alpha^{*} T_{0}}{\rho_{0}^{2}} \exp \left(-\beta^{*} t^{*}\right) \int_{-\infty}^{t^{*}} \exp \left(\beta^{*} t^{*}\right) \rho_{1}^{\prime 2}\left(t^{*}, x^{*}\right) \mathrm{d} t^{*},
$$

where $\alpha^{*}=\alpha / \omega, \beta^{*}=\beta / \omega, t^{*}=\omega t$ are dimensionless quantities, $x^{*}=x \omega / u_{\infty}$ or $x^{*}=x \omega / u_{0}$. In the case of high-frequency sound $\left(\beta^{*} \ll 1\right)$, Eq. (36) takes the form

$$
T_{3}^{\prime} \approx \frac{\alpha^{*} T_{0}}{\rho_{0}^{2}} \int_{-\infty}^{t^{*}} \rho_{1}^{\prime 2}\left(t^{*}, x^{*}\right) \mathrm{d} t^{*}
$$

and in the case of low-frequency sound $\left(\beta^{*} \gg 1\right)$ it rearranges in the equality as follows:

$$
T_{3}^{\prime} \approx \frac{\alpha^{*} T_{0}}{\beta^{*} \rho_{0}^{2}} \rho_{1}^{\prime 2}\left(t^{*}, x^{*}\right) .
$$

The main conclusion is that change of temperature caused by the low-frequency sound is much less effective than by the high-frequency one.

\section{Conclusions}

An exoteric chemical reaction results not only in a linear amplification $\left(B_{\infty}>0\right)$ or attenuation of sound itself $\left(B_{\infty}<0\right)$ but essentially influences a character of nonlinear interaction of sound and non-acoustic modes. As for the thermal mode, an excess temperature, associated with it, always grows in the standard attenuating fluids. Irreversibility of a chemical reaction $\left(B_{\infty}>0\right)$ leads not only to sound amplification, which never occurs in the Newtonian fluids, but to cooling of media of sound propagation. The efficiency of heating or cooling by the low-frequency sound is low. The linear absorption of the low-frequency sound itself is also weak. These two last properties look similar in a standard flow over a Newtonian fluid. The numerical evaluations for some waveforms of the equal energy reveal independence of acoustic heating (or cooling) on a concrete waveform. As for the chemical mode, this non-wave mode exists due to presence of a chemical reaction. The characteristic time of chemical reaction depends on $Q_{\rho}$ and $Q_{T}$, it does not longer equal $\tau_{\mathrm{c}}$, see Eq. (34) in the low-frequency domain and Eq. (35) in the high-frequency domain.

In this study, we did not consider neither standard attenuation nor thermal conductivity of a gas. All nonlinear effects of sound occur exclusively due to the presence of exoteric chemical reaction. Though small in the frames of this study, these corrections enlarge $\tau_{\mathrm{c}}$ or make it smaller. The equations governing an excess temperature attributable to the chemical mode, look similarly for both cases of low-frequency or high-frequency sound. A sign of an excess temperature depends on $Q_{\rho}$ and $Q_{T}$ for the high-frequency sound and on $Q_{T}$ exclusively for the low-frequency sound. Possibilities to control ambient temperature is much poor by the low frequency sound as compared with the high-frequency one. 
The domain of sound frequencies corresponding to low-frequency, or high-frequency regime, depends obviously on a concrete reaction. For the branching reactions such as $\mathrm{SiH}_{3} \rightarrow$ products, or $\mathrm{H} \rightarrow$ products, the characteristic duration of reaction is of order of $10^{-3} \mathrm{~s} \mathrm{[11]}$.

\section{References}

[1] O.V. Rudenko, S.I. Soluyan, Theoretical Foundations of Nonlinear Acoustic, Plenum, New York 1977.

[2] S. Makarov, M. Ochmann, Acustica 82, 579 (1996).

[3] A. Perelomova, Acta Acust. united with Acustica 89, 754 (2003).

[4] A. Perelomova, Phys. Lett. A 357, 42 (2006).
[5] A. Perelomova, Acta Acust. united with Acustica 96, 43 (2010).

[6] N.E. Molevich, Acoust. Phys. 48, 209 (2002).

[7] N.E. Molevich, Acoust. Phys. 49, 229 (2003).

[8] B. Riemann, Abhandl. Ges. Wiss. Göttingen, Math.-Physik. 8, 43 (1858-1859); repinted in The Collected Works of Bernard Riemann, Dover, New York 1953, p. 156.

[9] N.E. Molevich, Acoust. Phys. 48, 209 (2002).

[10] A. Perelomova, W. Pelc-Garska, Cent. Eur. J. Phys. 8, 855 (2010).

[11] M. Koshi, N. Nishida, T. Murakami, H. Matusi, J. Phys. Chem. 97, 4473 (1993). 